TITLE:

\title{
Simultaneous observation of nascent plasma and bubble induced by laser ablation in water with various pulse durations
}

\author{
$\operatorname{AUTHOR}(\mathrm{S}):$ \\ Tamura, Ayaka; Matsumoto, Ayumu; Fukami, \\ Kazuhiro; Nishi, Naoya; Sakka, Tetsuo
}

\section{CITATION:}

Tamura, Ayaka ... [et al]. Simultaneous observation of nascent plasma and bubble induced by laser ablation in water with various pulse durations. Journal of Applied Physics 2015, 117(17): 173304.

\section{ISSUE DATE:}

2015-05-06

URL:

http://hdl.handle.net/2433/200908

\section{RIGHT:}

(C) 2015 American Institute of Physics. This article may be downloaded for personal use only. Any other use requires prior permission of the author and the American Institute of Physics. 


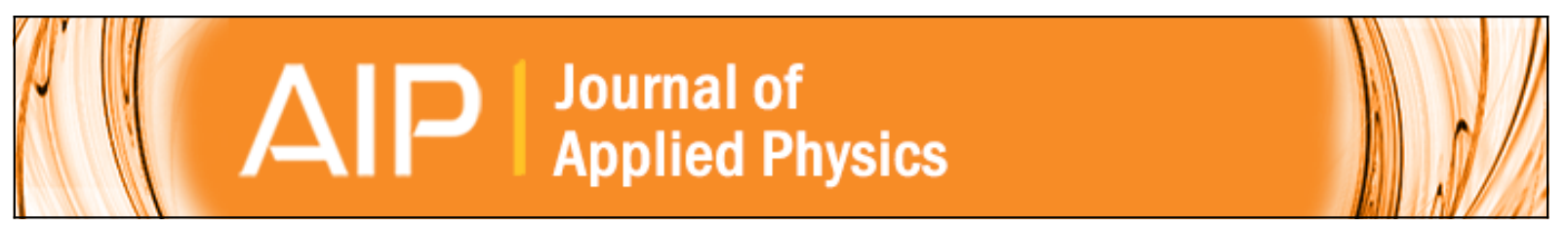

\section{Simultaneous observation of nascent plasma and bubble induced by laser ablation in water with various pulse durations}

Ayaka Tamura, Ayumu Matsumoto, Kazuhiro Fukami, Naoya Nishi, and Tetsuo Sakka

Citation: Journal of Applied Physics 117, 173304 (2015); doi: 10.1063/1.4919729

View online: http://dx.doi.org/10.1063/1.4919729

View Table of Contents: http://scitation.aip.org/content/aip/journal/jap/117/17?ver=pdfcov

Published by the AIP Publishing

\section{Articles you may be interested in}

Low-density plasma formation in aqueous biological media using sub-nanosecond laser pulses

Appl. Phys. Lett. 105, 063701 (2014); 10.1063/1.4892665

Nanoparticle formation in a cavitation bubble after pulsed laser ablation in liquid studied with high time resolution small angle $x$-ray scattering

Appl. Phys. Lett. 101, 103104 (2012); 10.1063/1.4750250

Modeling the time evolution of laser-induced plasmas for various pulse durations and fluences

Phys. Plasmas 11, 2182 (2004); 10.1063/1.1691454

Relaxing melt and plasma bubbles in laser-pulsed metals

J. Appl. Phys. 94, 6280 (2003); 10.1063/1.1619570

Starting mechanisms and dynamics of bubble formation induced by a Ho:Yttrium aluminum garnet laser in water J. Appl. Phys. 84, 5905 (1998); 10.1063/1.368906

\section{AIP $\left.\right|_{\text {APL Photonics }}$}

APL Photonics is pleased to announce Benjamin Eggleton as its Editor-in-Chief

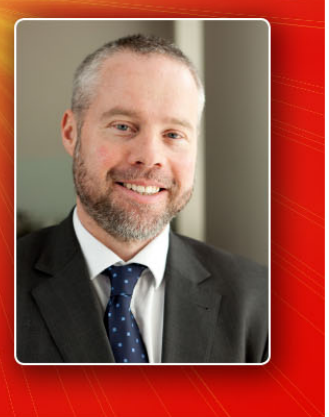




\title{
Simultaneous observation of nascent plasma and bubble induced by laser ablation in water with various pulse durations
}

\author{
Ayaka Tamura, ${ }^{1, a)}$ Ayumu Matsumoto, ${ }^{1}$ Kazuhiro Fukami, ${ }^{2}$ Naoya Nishi, ${ }^{1}$ \\ and Tetsuo Sakka, ${ }^{1, b}$ \\ ${ }^{1}$ Department of Energy and Hydrocarbon Chemistry, Graduate School of Engineering, Kyoto University, \\ Kyoto 615-8510, Japan \\ ${ }^{2}$ Department of Materials Science and Engineering, Graduate School of Engineering, Kyoto University, \\ Kyoto 606-8501, Japan
}

(Received 19 February 2015; accepted 23 April 2015; published online 6 May 2015)

\begin{abstract}
We investigate the effects of pulse duration on the dynamics of the nascent plasma and bubble induced by laser ablation in water. To examine the relationship between the nascent plasma and the bubble without disturbed by shot-to-shot fluctuation, we observe the images of the plasma and the bubble simultaneously by using two intensified charge coupled device detectors. We successfully observe the images of the plasma and bubble during the pulsed-irradiation, when the bubble size is as small as $20 \mu \mathrm{m}$. The light-emitting region of the plasma during the laser irradiation seems to exceed the bubble boundary in the case of the short-pulse (30-ns pulse) irradiation, while the size of the plasma is significantly smaller than that of the bubble in the case of the long-pulse (100-ns pulse) irradiation. The results suggest that the extent of the plasma quenching in the initial stage significantly depends on the pulse duration. Also, we investigate how the plasma-bubble relationship in the very early stage affects the shape of the atomic spectral lines observed at the later delay time of $600 \mathrm{~ns}$. The present work gives important information to obtain high quality spectra in the application of underwater laser-induced breakdown spectroscopy, as well as to clarify the mechanism of liquid-phase laser ablation. (C) 2015 AIP Publishing LLC.
\end{abstract}

[http://dx.doi.org/10.1063/1.4919729]

\section{INTRODUCTION}

Laser ablation of solid target in water is a phenomenon which is key to many practical applications, ${ }^{1}$ such as elemental analysis of solid targets in water, ${ }^{2-7}$ direct generation of nanoparticles in water, ${ }^{8,9}$ and laser peening of solid materials. $^{10,11}$ Especially, we focus on the underwater laserinduced breakdown spectroscopy (LIBS), which is based on the emission spectroscopy of laser plasma. LIBS can be applied to various solid targets in various environments, e.g., rocks at sea bottoms for resource exploration. ${ }^{12-18}$ To achieve the underwater in situ analysis, it is necessary to generate a plasma suitable for the elemental analysis in a water-confined geometry.

In general, the laser ablation plasma in water is confined strongly to a small volume, ${ }^{19,20}$ which results in a highdensity state of plasma, and hence, suffers from spectral broadening of the atomic emission lines and an intense continuous spectrum. ${ }^{21,22}$ It is difficult to obtain spectral lines narrow enough to be applied to in situ underwater elemental analysis, as compared with LIBS in gas or air environments. $^{23,24}$ Also, exploration for seabed mineral resources needs the analytical scheme which can be used under high pressure. Double-pulse irradiation scheme is known to give narrow spectral lines at ambient pressure, ${ }^{2-5,23-25}$ but does not work under high pressures corresponding to $500 \mathrm{~m}$ depth or deeper. ${ }^{26,27}$ On the other hand, a long single pulse, such as

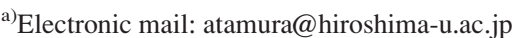

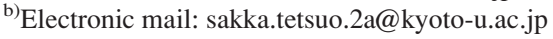

$100 \mathrm{~ns}$ or longer, ${ }^{28}$ gives narrow spectral lines even under high-pressure water environment. ${ }^{17}$ Recently, underwater LIBS experiments at high pressure about $30 \mathrm{MPa}$, corresponding to the depths of about $3000 \mathrm{~m}$, have been also performed for the application to the resource exploration at sea bottoms. ${ }^{14-18}$

The mechanism of the emission of narrow spectral lines in the case of the long-pulse irradiation seems to be attributed to the timing of the bubble generation. ${ }^{29}$ When a short pulse is employed as a laser source, the bubble does not seem to sufficiently expand during the pulse, and then the plasma inside the bubble cannot be expanded. On the other hand, the timing of the bubble generation by the long-pulse irradiation is very early in the rising edge of the pulse, so that the remaining part of the pulse energy can be used for the direct heating of the plasma. However, the relationship between the plasma and bubble expansions is not fully understood, e.g., the timing of the plasma generation relative to the bubble generation, and the size of the plasma relative to the bubble. Also, the quenching mechanism of the plasma in water is not fully clarified. The difference between the short-pulse and the longpulse irradiations should be discussed from the point of view of the dynamics of the plasma and the bubble. In comparing precisely the dynamics of the plasma and the bubble with the long-pulse or the short-pulse irradiation, the pulse-to-pulse instability of the emission spectra and optical emission images cause serious troubles. Especially in the case of the long-pulse irradiation, the timing of the plasma generation and the intensity of the optical emission, as well as the timing of the bubble generation, fluctuate pulse-to-pulse. To overcome 
these difficulties caused by the pulse-to-pulse instability, it is effective to study the initial process of this phenomenon by observing the plasma and the bubble at the same time with a single shot irradiation. It has been reported in our previous work $^{29}$ that a very nascent bubble as well as the plasma emerges during the irradiation of the laser pulse, regardless of the pulse duration, namely, $20 \mathrm{~ns}$ or $150 \mathrm{~ns}$, and the size of the bubble or the plasma is as small as 20 to $30 \mu \mathrm{m}$ in diameter or less. Since the position of the bubble generation fluctuates pulse-to-pulse by 10 to $20 \mu \mathrm{m}$ in the lateral direction, the plasma image and the bubble image obtained with different pulse shots cannot be compared with a high precision. Additionally, we note that nanoparticles seem to be produced in the initial stage of the liquid-phase laser ablation process. ${ }^{30}$ It is also very important to investigate the relationship between the nascent plasma and bubble for the understanding of nanoparticle production.

In the present work, we observe the plasma image and the bubble image at the same time with a single laser shot, which enables us to get rid of the position error caused by the pulse-to-pulse fluctuation mentioned above. We examine the effect of pulse duration on the relationship between the nascent plasma and bubble at the very initial stage of the underwater laser ablation phenomenon. We discuss how these differences in the initial stage cause the difference in the spectral feature observed in the later stage, in which the LIBS measurement is actually performed.

\section{EXPERIMENTAL}

Experimental setup for the simultaneous measurement of the bubble image by shadowgraphy and the optical emission image is shown in Fig. 1. A home-built flashlamppumped Q-switched Nd:YAG laser oscillating at the fundamental wavelength of $1064 \mathrm{~nm}$, the pulse energy of $1.8 \mathrm{~mJ}$, and the repetition rate of $0.3 \mathrm{~Hz}$ was used. The pulse duration was controlled by the procedure given in our previous publication, ${ }^{28}$ and was 30,50 , or $100 \mathrm{~ns}$. The laser beam was focused onto a target surface from the direction normal to the surface by a $70 \mathrm{~mm}$ focal-length plano-convex lens. A $\mathrm{Cu}$ plate (Nilaco, CU-113421) used as a target was placed in a quartz cell filled with ultrapure water (Millipore, Milli-Q Gradient). The depth of the water at the target surface is about $10 \mathrm{~mm}$. The laser irradiation spot was illuminated by a 532-nm laser beam, which was split from the original laser by the polarizing beam splitter (PBS) and passed through a nonlinear crystal for the second harmonic generation (KTP). After the crystal, the laser beam was introduced to a speckle killer (Nanophoton, SK-11-Mg-5) to reduce speckle noises. We prolonged the optical path of the original beam, which is used as an ablation laser, to ensure that the trigger signal from the laser pulse is early enough to drive the detection system during the pulse, as well as to observe the nascent plasma and bubble with intense back illumination. The plasma and bubble produced by the ablation in water were observed in the direction parallel to the target surface. A $50 \times$ microscope objective lens (Mitutoyo, G Plan Apo 50×) and an imaging lens (Mitutoyo, MT-4) were used as imaging optics. After passing through these optics, the plasma emission and laser back illumination were split to two beams by the BS and imaged on the imaging plane of the two intensified CCDs (ICCDs) (Roper Scientific, PI-MAX: $1 \mathrm{~K}$ ). One ICCD measured the shadowgraphs for the images of the bubble, and the other measured the optical emission images of the plasma. We need to remove intense plasma emission from the shadowgraph to clearly observe the image of the bubble. Therefore, we placed an interference filter $(532 \mathrm{~nm})$ in the optical path between the objective lens and the ICCD for shadowgraphy, and ensured that only the light of the wavelength of $532 \mathrm{~nm}$ is detected. On the other hand, we need to remove the back illumination for observing only the plasma emission image. Therefore, a notch filter $(532 \mathrm{~nm})$ was placed in the optical path between the objective lens and the ICCD, in this case, to block the wavelength of $532 \mathrm{~nm}$. The plasma and bubble images were observed at the three delay times during the laser irradiation. The first and third delay times were set so that the instantaneous intensity of the pulse is almost half of that at the peak in the earlier and later parts of the peak, respectively. The second delay time was set almost at the peak of the pulse. The gate width of the ICCD was set to $10 \mathrm{~ns}$.

The emission spectra were measured in the direction parallel to the target surface. The optical emission was focused into an optical fiber bundle (Oriel Instruments, 77532) through two $60 \mathrm{~mm}$ focal-length plano-convex lenses. The end of the fiber was connected to the spectrograph (Bunkoukeiki, MK-302) equipped with an ICCD (Princeton Instruments, ICCD1024MTDGE/1). The slit width and the groove density of the diffraction grating used in the spectrograph are $100 \mu \mathrm{m}$ and

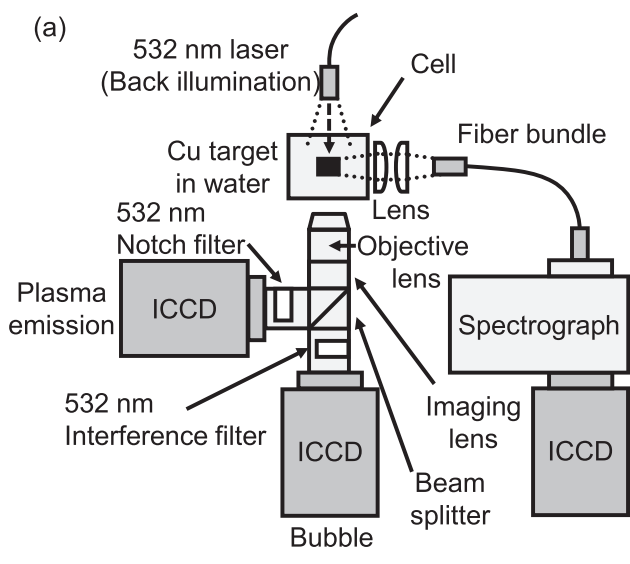

FIG. 1. (a) Experimental setup for the simultaneous measurement of the shadowgraph image and the emission image, and the measurement of emission spectrum. (b) Schematic illustration of the optical path of laser beam for the irradiation of the target and the back illumination. 
Plasma emission Shadowgraph

(a) Pulse duration: 30 ns

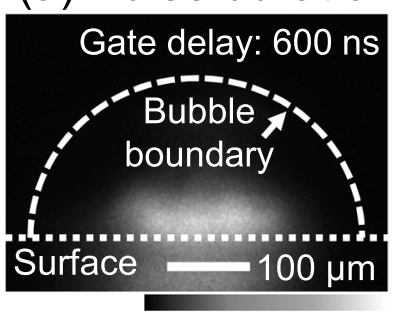

75

(b) $50 \mathrm{~ns}$

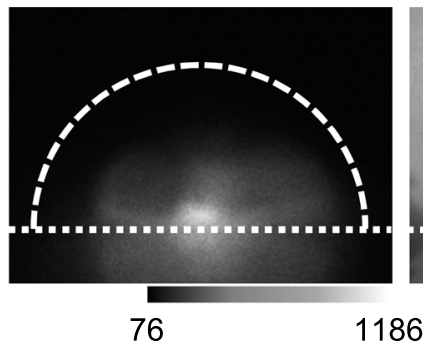

1224

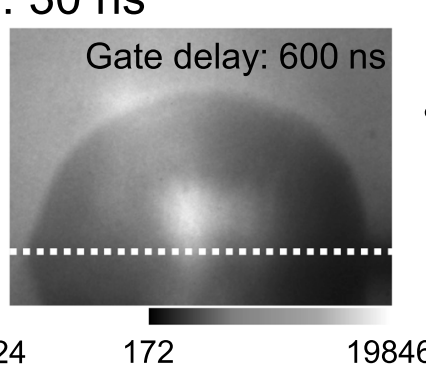

172

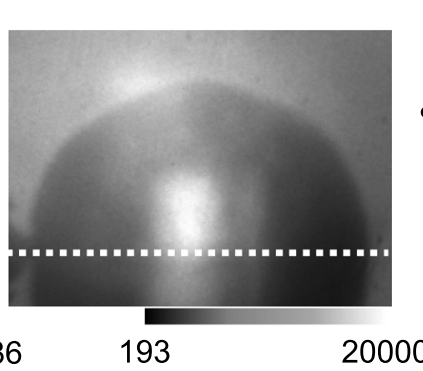

(c) $100 \mathrm{~ns}$
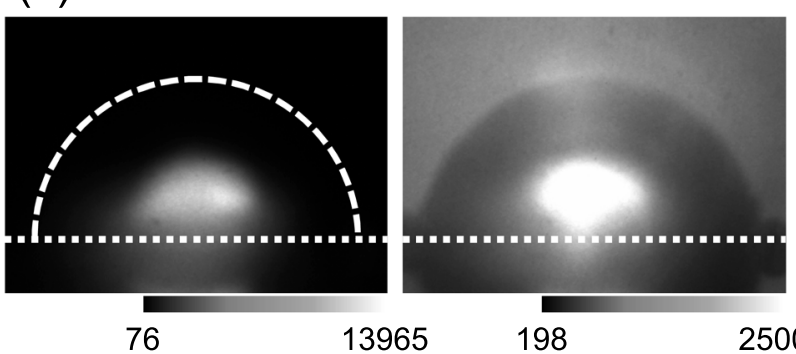

198

25000

1200 grooves/mm, respectively. The emission spectra were measured at the delay time of $600 \mathrm{~ns}$ after the laser irradiation. The gate width of the ICCD was set to $1000 \mathrm{~ns}$. The trigger level was set to the rising edge of the laser pulse. We observed spectral lines of $\mathrm{Cu}$ I 324.8 and $327.4 \mathrm{~nm}$. Since the lower level for these lines is the ground state, the emission can be re-absorbed by abundant ground state atoms, and therefore the spectral lines suffer from the strong self-absorption. ${ }^{31-34}$ The self-absorption effect becomes stronger with increase in the population density of the lower level of the transition, and it is a significant problem that makes underwater LIBS difficult since the strong confinement by the water phase causes high population density especially for the ground state. Therefore, the choice of these spectral lines allows us a severe test for the effects upon the spectral deformation. We also observed the plasma and bubble emission after $600 \mathrm{~ns}$ from the laser irradiation.

\section{RESULTS AND DISCUSSION}

Figure 2 shows plasma emission images, shadowgraph images, and emission spectra obtained after $600 \mathrm{~ns}$ from the laser irradiation. The pulse durations of 30,50 , or $100 \mathrm{~ns}$ are employed. Narrow spectral lines were obtained when the pulse duration is the longest, namely, $100 \mathrm{~ns}$. As shown in the plasma emission images, plasma emission intensity obtained by the 100-ns pulse is higher than that obtained by the $30-n s$ and $50-n s$ pulses. The bubble sizes obtained by the different

\section{Spectra}

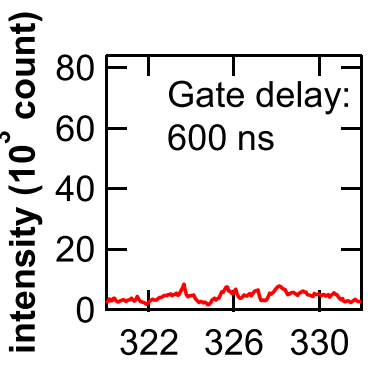

FIG. 2. Plasma emission images (left column), shadowgraph images (center column), and emission spectra (right column) obtained after $600 \mathrm{~ns}$ from the laser irradiation. The pulse durations of (a) 30 , (b) 50 , or (c) $100 \mathrm{~ns}$ were employed. In plasma emission images, the bubble boundary is indicated by a broken line.

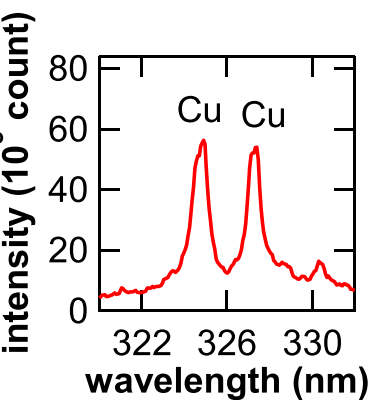

pulse durations are almost the same after $600 \mathrm{~ns}$ from the pulse irradiation. The emission is observed below the target surface in the images. This is a reflection of light at the target surface. It is difficult to observe the optical emission from the plasma at the direction exactly parallel to the target surface.

Figure 3 shows the results obtained by the irradiation of the 30-ns pulse. In Fig. 3(a), the temporal profile of the 30-ns pulse and the gating profile of the ICCD detector for the measurement of plasma emission images and shadowgraph images are indicated. Here, three different gate timings, A, B, and C are shown in dotted lines color-coded in different colors. In Figs. 3(b) and 3(c), the plasma emission images and the shadowgraph images are shown, respectively. The plasma emission images and shadowgraph images in these figures were observed at the gate timing shown in Fig. 3(a). In the case of the irradiation with the 50-ns and 100-ns pulse, the results of plasma emission images and shadowgraph images are shown in Figs. 4 and 5, respectively, in the same manner as in Fig. 3.

As shown in Fig. 3(c), the bubble at the gate timing $\mathrm{A}$ is hard to identify due to a small obscure shadow. We think that this shadow is assigned to a shock wave, since the expansion rate of the shadow is $\sim 2000 \mathrm{~m} \cdot \mathrm{s}^{-1}$, which is comparable to the speed of sound in water $\left(\sim 1500 \mathrm{~m} \cdot \mathrm{s}^{-1}\right)$. We can see the obscure annular band in Figs. 3(b) and 3(c), which is assigned to the shock wave. The reason that the shock wave has such a wide width is not clarified. This is probably because the shock wave propagates hemi- 
(a) Gate timing

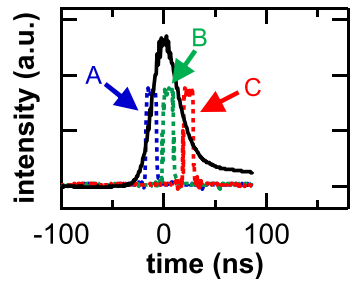

(b) Plasma emission (c) Shadowgraph
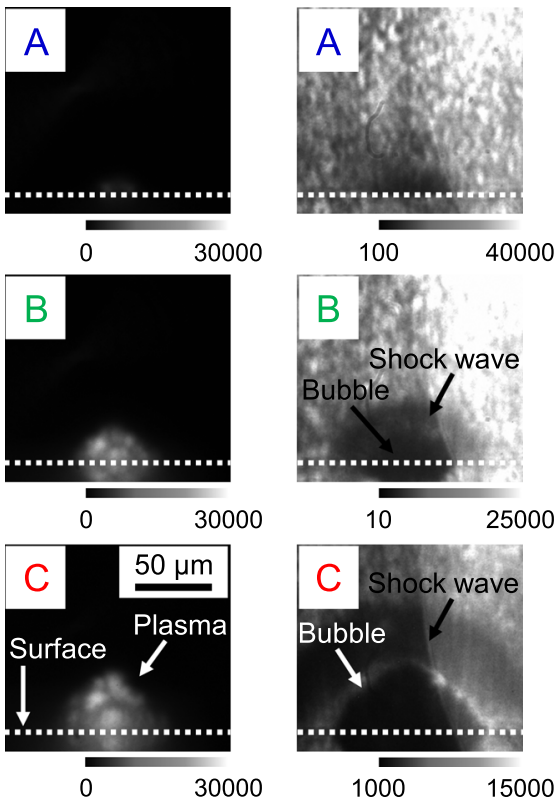

FIG. 3. (a) Temporal profile of the 30-ns pulse, and the gating profile of the ICCD detector for the measurement of plasma emission images and shadowgraph images, (b) plasma emission images, and (c) shadowgraph images. The results were obtained by the irradiation of the 30-ns pulse. The plasma emission images and bubble images indicated by A, B, and C were observed at the corresponding gate timing shown in (a).

spherically, and we observed the region with a low refractive index behind the shock wave front due to low pressure when the hemisphere is very small. Also, the fast speed of the shock wave at the initial stage and the ICCD gate width of 5 ns may cause the width of shock wave. In the shadowgraph representing the gate timing $\mathrm{B}$, we can recognize a dark shadow of the bubble in the obscure shadow caused by the shock wave. The plasma emission region and the bubble expand with time. Although the bubble at the gate timing A is hard to identify, we can observe the plasma emission at this timing (weak emission can be seen, if looking at the digital image of Fig. 3(b) very carefully).

Figure 4 shows the plasma emission images and the shadowgraph images obtained by the irradiation of the 50-ns pulse. The bubble at the gate timing $\mathrm{A}$ is also hard to identify due to an obscure shadow. Again, we think that this shadow is assigned to a shock wave. On the other hand, the bubble at the gate timing $\mathrm{B}$ can be clearly recognized in the shadow caused by the shock wave. The size of the plasma emission region at the peak of the pulse, which corresponds to the gate timing B, seems to be the same as that of the bubble seen in the shadowgraph (see the center image of Fig. 4(c)).

Figure 5 shows the plasma emission images and the shadowgraph images obtained by the irradiation of the 100- (a) Gate timing

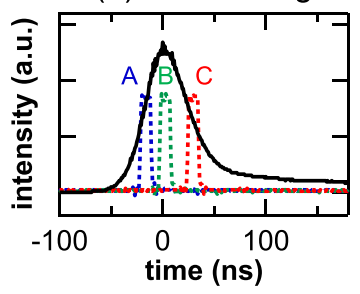

(b) Plasma emission (c) Shadowgraph

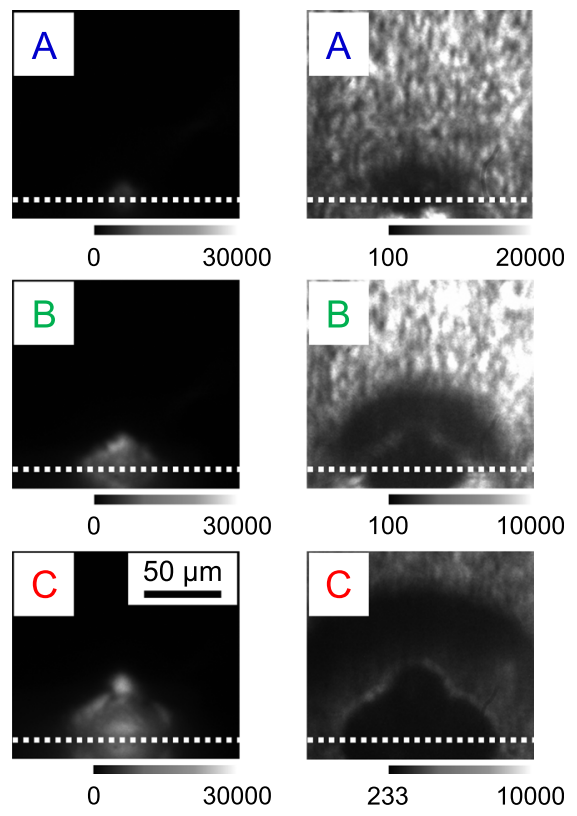

FIG. 4. (a) Temporal profile of the 50-ns pulse, and the gating profile of the ICCD detector for the measurement of plasma emission images and shadowgraph images, (b) plasma emission images, and (c) shadowgraph images. The results were obtained by the irradiation of the 50-ns pulse. The plasma emission images and bubble images indicated by $\mathrm{A}, \mathrm{B}$, and $\mathrm{C}$ were observed at the corresponding gate timing shown in (a).

ns pulse. The bubble can be recognized in the obscure shadow caused by the shock wave even at the gate timing A. The size of the plasma emission region seems to be smaller than that of the bubble at any gate timing. Here, we define the plasma emission region to be the region where the image is white enough, or more precisely, the intensity is more than $1 / 10$ of the maximum intensity in the plasma.

In order to compare the size of the plasma emission region and bubble more precisely, we have plotted the intensity profile in the plasma emission image and the shadowgraph image along the line normal to the target surface passing through the center of the plasma or the bubble. The results are shown in Fig. 6. The origin of each graph in Fig. 6 is the position of the target surface. The value of the intensity profile was averaged over the 10 adjacent pixels in the image. In this figure, we have employed the plasma emission images and the shadowgraph images at the gate timing $\mathrm{B}$ of each series of the measurement done by irradiating the laser pulse with different pulse duration, namely, the 30-ns, 50-ns, and 100-ns pulses. The red dotted lines in Fig. 6 show the intensity profiles in the plasma emission images. The blue solid lines in Fig. 6 show the intensity profiles in the shadowgraph. The intensity profile of the shadowgraph is low in the region of the bubble due to the intense scattering of the back 
(a) Gate timing

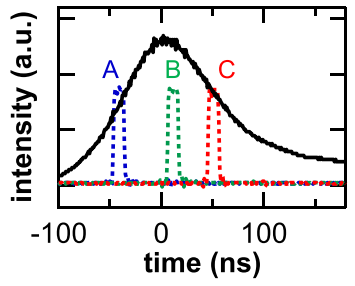

(b) Plasma emission (c) Shadowgraph
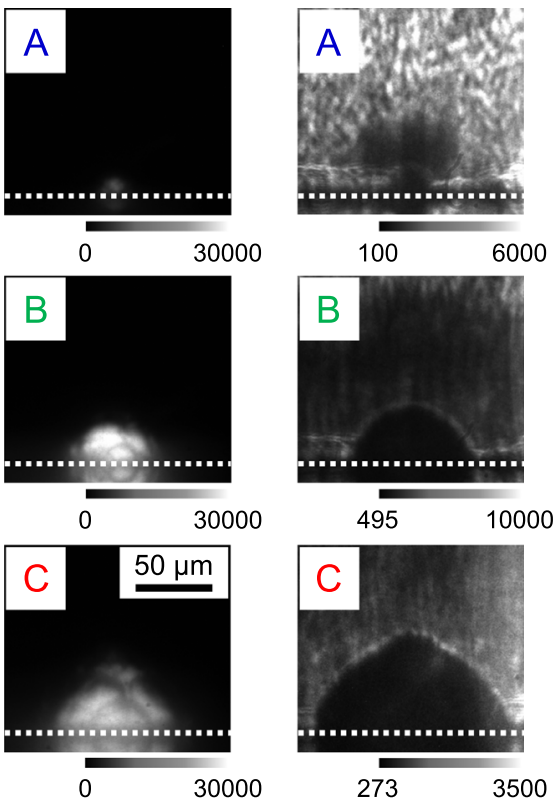

FIG. 5. (a) Temporal profile of the 100-ns pulse, and the gating profile of the ICCD detector for the measurement of plasma emission images and shadowgraph images, (b) plasma emission images, and (c) shadowgraph images. The results were obtained by the irradiation of the 100-ns pulse. The plasma emission images and bubble images indicated by A, B, and C were observed at the corresponding gate timing shown in (a).

illumination laser. It should be noted that the dark region appears also due to the shadow of the shock wave, which can be noticed in the shadowgraph profile just outside the bubble, as seen in Fig. 6. Therefore, we assign the leftmost peak of the blue solid line to be the bubble boundary. The intensity of the plasma emission profile falls in the vicinity of the bubble boundary. The distance from the bubble boundary to the position where the plasma emission intensity is half its maximum value is defined as the spacing between the plasma and the bubble boundary. By comparing the cases of 50 and $100 \mathrm{~ns}$, it can be seen that the spacing increases from $\sim 5$ to $\sim 15 \mu \mathrm{m}$ with increasing the pulse duration. In consequence, in the case of the irradiation with the 30-ns pulse, it is clearly demonstrated not only by visually examining the image but also by the numerical plot of the intensity profiles that the size of the bubble is smaller than that of the plasma emission region. As supporting results, we show the intensity profiles normal to the target surface at the gate timings $\mathrm{A}$ and $\mathrm{C}$ in Figs. S1 and S2 in supplementary material, respectively. ${ }^{35}$ As shown in Fig. S1, it is difficult to distinguish the shock wave and the bubble at the gate timing A, especially for the case of short pulses. On the other hand, at the gate timing $\mathrm{C}$, we can see that the plasma emission region is smaller than the bubble as shown in Fig. S2. We also show the intensity (a) Pulse duration: 30 ns

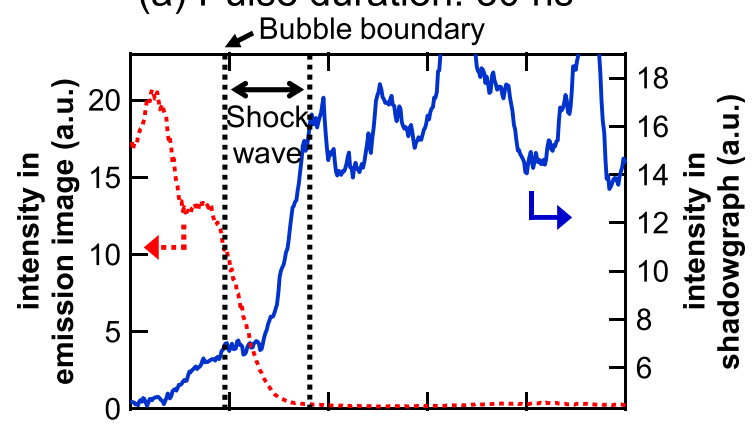

(b) $50 \mathrm{~ns}$

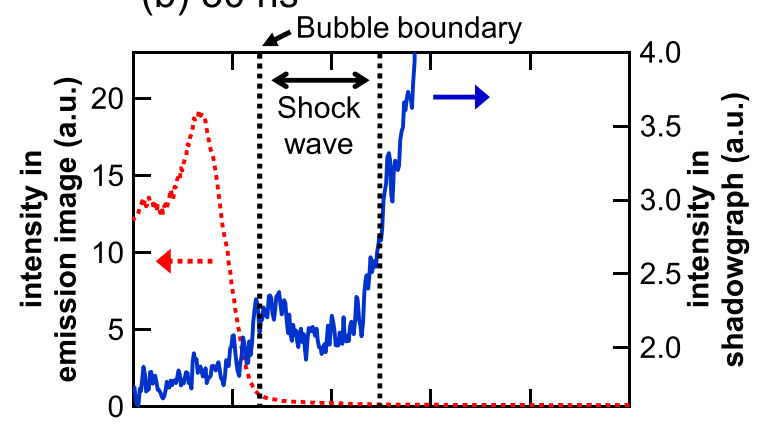

(c) $100 \mathrm{~ns}$

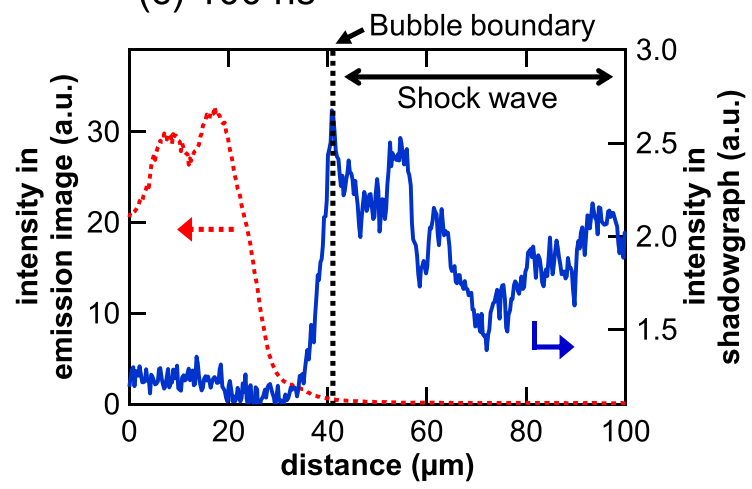

FIG. 6. Intensity profile in the plasma emission image (red dotted line) and the shadowgraph image (blue solid line) along the line normal to the target surface passing through the center of the plasma or the bubble at the gate timing of B in Figs. 3-5. The pulse durations of (a) 30, (b) 50, or (c) $100 \mathrm{~ns}$ were employed.

profiles along the line parallel to and $13 \mu \mathrm{m}$ above the target surface at the gate timing $\mathrm{B}$ in Fig. 7. According to Figs. 7(b) and 7(c), we can see the same tendency as the results shown in Figs. 6(b) and 6(c). In the case of the irradiation with the 30-ns pulse (Fig. 7(a)), however, it is difficult to determine the boundary of the bubble due to the shadow of the shock wave.

In the following, we discuss the effect of pulse duration upon the shape of the spectral lines from the point of view of the interrelationship between the nascent plasma and bubble at the same timing. In the case of the irradiation with the short pulses, like the 30-ns pulse, the size of the plasma during the laser irradiation is larger than that of the bubble. There are two possible explanations. One is that the excited species ejected from the surface go outside the bubble, and keep emitting light. The other is that the water surrounding the bubble is scattering the intense emission from the plasma inside the bubble. Even if the plasma is inside the bubble, 
(a) Pulse duration: 30 ns

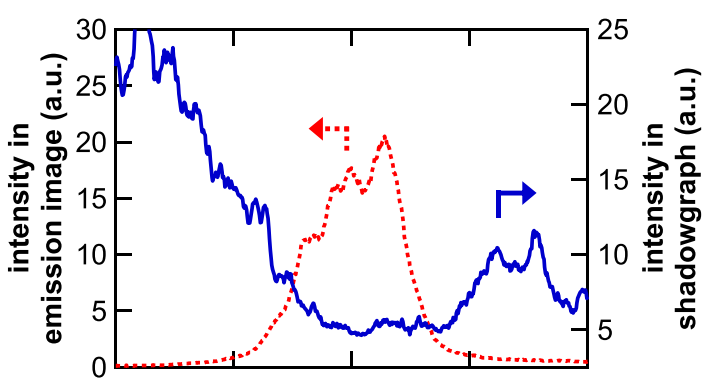

(b) $50 \mathrm{~ns}$

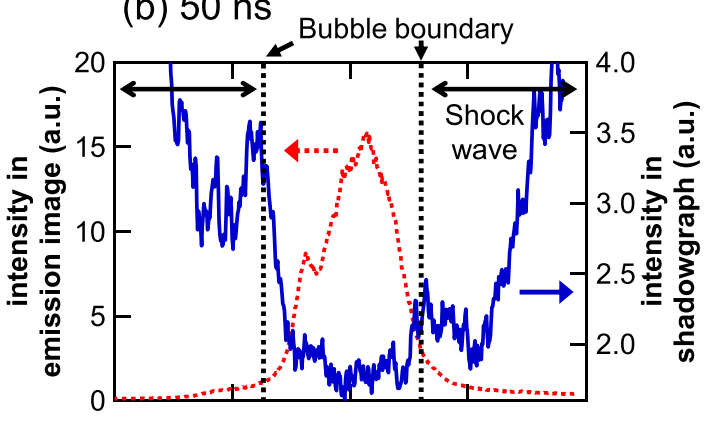

(c) $100 \mathrm{~ns}$

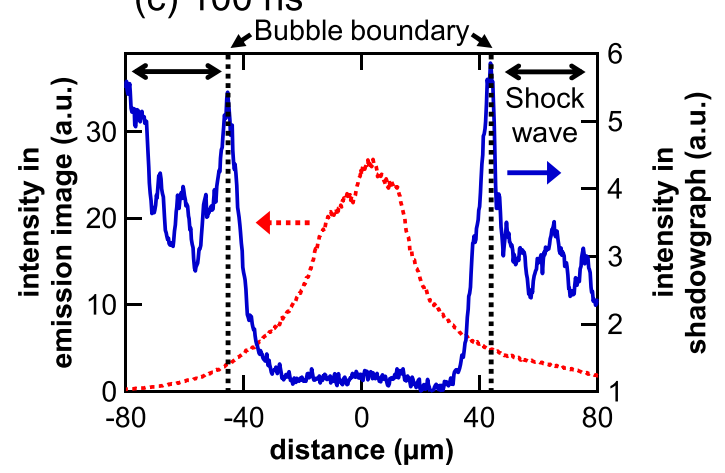

FIG. 7. Intensity profile in the plasma emission image (red dotted line) and the shadowgraph image (blue solid line) along the line parallel to and $13 \mu \mathrm{m}$ above the target surface at the gate timing of B in Figs. 3-5. The pulse durations of (a) 30 , (b) 50, or (c) 100 ns were employed.

the emission from the plasma illuminates the surrounding water, may be scattered by the water, and detected in the image. The direct emission from the plasma plus the scattered light from the surrounding water might make the plasma seemingly larger than the bubble. However, we see in the middle image of Fig. 5(b) that the scattering from the surrounding water is not observed when the long pulse is used, although the emission from the plasma is more intense than that in the case of short-pulse irradiation shown in Fig. 3 (b) in which we clearly see emission from the surrounding water. Therefore, the former explanation seems to be more likely. According to the former explanation, the ablated species directly interact with water, and they must be rapidly quenched. It should be noted that the whole pulse energy is supplied in a short time period, and the bubble size during the energy supply is very small. It should also be noted that the bubble expansion speed is not strongly dependent on the pulse duration. ${ }^{36}$ Therefore, the plasma expansion speed exceeds the bubble expansion and the excited species penetrate into water from the bubble. Such direct interaction of plasma and water results in a rapid quenching of the plasma.
At the later delay time $(600 \mathrm{~ns})$, when the density becomes low enough and the emission spectra are observed, the emission intensity has already decayed into very low level, due to the intense quenching in the early stage of the plasma and bubble formation. The emission spectra observed with the short pulse depicted in Fig. 2(a) show the noise level spectra in the spectral range where the intense emission from $\mathrm{Cu}$ atoms is expected.

On the other hand, the size of the plasma is sufficiently smaller than that of the bubble during the pulse, when the 100 -ns pulse is used. This is probably due to relatively gradual supply of the energy by the long-pulse irradiation, where the leading edge of the pulse produces the plasma as well as the bubble, and the successive pulse energy is absorbed by the plasma in the expanding bubble without ablation of the target surface. It should be noted that the plasma residing far from the bubble boundary is not quenched directly by the liquid phase water. This means that the quenching of the plasma in a large bubble should be suppressed. In the case of long pulse, the plasma is excited not only at the timing when the bubble is produced but also when the bubble has already grown. Such condition leads to the longer persistence of the plasma. Also, in such condition the plasma not only expands but also keeps a comparatively high emission intensity even after a long delay time when the density becomes low enough and the LIBS measurements will be made. As a result, the emission spectra obtained with the long-pulse irradiation depicted in Fig. 2(c) show the narrow spectral lines with high intensity.

In our previous work, ${ }^{29}$ we found that the damage to the sample surface seems to be minimal by using the long pulse, although the long pulse gives intense spectral lines. The damage by the short-pulse irradiation is significantly more severe than that by the long-pulse irradiation. ${ }^{29}$ These results also support that the later part of the pulse energy of the long pulse is absorbed by the plasma, while the large part of the short pulse irradiates the target surface directly. In other words the long-pulse irradiation results in the mild ablation and the persistent energy supply to the plasma. These are the reasons that narrow spectral lines can be obtained by the long-pulse irradiation.

As shown in Figs. 3, 4, and 5, the contour shape of the plasma emission region seems to correspond to that of the bubble. By comparing the shape of the plasma and the bubble, we can notice that the position of a bump seen in the plasma image is located exactly at the same position of the bump of the bubble. This is most clearly seen in the images of the plasma and bubble shown in the lowermost row of Figs. 4(b) and 4(c). While the shape of the nascent bubble surface seems to reflect the shape of the plasma periphery, the bubble shape at the delay time of 600 ns does not reflect the plasma shape anymore, as is seen in Fig. 2. In Fig. 2(a), we can see flat-shaped plasma with relatively low intensity, while the bubble is a relatively large hemisphere with a smooth boundary.

The strong correlation between the shape of the plasma and that of the bubble suggests that the edge of the plasma is in contact with water, or at least that the ablated species in the plasma strongly interact with the boundary of the bubble. 
In other words, in the early stage during the laser pulse irradiation, the bubble evolution is strongly correlated with the plasma evolution. The present results give important suggestion on the mechanism of the bubble formation, i.e., the bubble is formed and expands following the expansion of the plasma, at least in the case of short-pulse irradiation, where the size of the plasma, or more precisely the light emitting region, exceeds that of the bubble at the timing of the pulse peak (see Fig. 6(a)). This leads to a practical idea that the bubble expansion can be controlled by the ablated species. In the case of the irradiation with the longer pulses, the portion of the ablated species is probably ejected into the water phase at the initial stage of the ablation, and this may be the reason for the correlation between the shape of the plasma and that of the bubble.

Ibrahimkutty et al. have shown that nanoparticle formation takes place inside the bubble. ${ }^{37}$ The strong interaction between the ablated species and the bubble boundary indicates that the plasma cooling starts from the external shells of the plasma. This might cause the constant temperature at the plasma core, which probably gives narrow size distribution of nanoparticles as Dell'aglio et al. have mentioned. ${ }^{30}$ On the other hand, the present results indicate that a portion of the species may penetrate the water phase immediately after the laser irradiation. It has been suggested that species originating in the water phase is introduced from the bubble boundary at the initial stage. ${ }^{38}$ Lam et al. have suggested the rapid oxidation of the ablated species in the plasma. ${ }^{39} \mathrm{We}$ consider that a strong interaction between the ablated species and the water causes the significant supply of oxygen from the water phase into the plasma.

As mentioned above, the shape of the nascent bubbles has some bumps. This is in contrast with the bubble after $600 \mathrm{~ns}$ from the laser irradiation. The presence of such bumps indicates that the bubble boundary is unstable. To minimize the interfacial energy, the bubble interface should be smooth. The smooth interface is actually observed at $600 \mathrm{~ns}$ from the laser irradiation, as is shown in Fig. 2. In Fig. 2(c), the bubble with a smooth hemispherical boundary as well as the hemispherical plasma is observed for the longpulse irradiation. At this delay time, the bubble expansion is not anymore caused by the interaction with the plasma, and hence, the plasma and bubble tend to take the most stable shape, without affecting each other.

The shape of the nascent plasma is also bumpy, as mentioned above. It seems that the bumps look like bright spots (see Figs. 3-5). This is probably because droplets are ejected from the target surface by phase explosion immediately after the laser irradiation, and they are hot enough to give bright spots. We consider that in the case of phase explosion, some droplets consisting of the target species with high temperature are separately ejected. The bright spots fluctuated shotby-shot. This is probably due to inhomogeneous ejection of the droplets. Currently, we consider that relatively large droplets are ejected from the target surface in the early stage during the laser irradiation. However, the bright spots disappear after $600 \mathrm{~ns}$ from the laser irradiation. The reason for the disappearance might be due to the evaporation of the droplets, resulting in smaller particles and atomic species.
Another explanation is that rapidly quenched droplets transferred to the water phase and disappeared from the plasma region. The latter is supported by the observation of $\mu \mathrm{m}$ scale large particles in water phase after the laser ablation of solid target in water. ${ }^{40}$

\section{CONCLUSIONS}

In the present work, we have investigated the effects of pulse duration on the dynamics of the nascent plasma and bubble produced by laser ablation in water. We have employed different pulse durations, namely, 30, 50, and 100 ns. Simultaneous measurement of the plasma emission image and the bubble image during the pulse irradiation has been successfully demonstrated in a single laser shot. The relationship between the size of the plasma and that of the bubble was quite different depending on the pulse durations. In the case of the irradiation with the short pulses, the size of the nascent plasma is larger than or similar to that of the nascent bubble. In the case of the irradiation with the long pulses, the size of the nascent plasma is significantly smaller than that of the nascent bubble. Such early dynamics of the plasma and the bubble suggest intense quenching of the plasma by direct interaction with liquid water in the case of the short-pulse irradiation. To obtain narrow spectral lines after a certain delay time, the expansion of the bubble during the initial stage of the ablation plays a key role. This is successfully accomplished by the long-pulse irradiation. By controlling the interrelationships between the nascent plasma and the bubble, the performance of the applications of the liquid-phase laser ablation will be greatly improved. The present results give important insight in the optimization of the laser parameters for underwater LIBS. Finally, we would like to emphasize that the present study significantly contributes to the basic understanding of liquid-phase laser ablation process.

\section{ACKNOWLEDGMENTS}

This work was supported by JSPS KAKENHI Grant Nos. 23560023, 13J04184, and 14J02461.

${ }^{1}$ G. W. Yang, Laser Ablation in Liquids: Principles and Applications in the Preparation of Nanomaterials (Pan Stanford Publishing, Singapore, 2012).

${ }^{2}$ A. De Giacomo, M. Dell'Aglio, F. Colao, and R. Fantoni, Spectrochim. Acta, Part B 59, 1431 (2004).

${ }^{3}$ A. De Giacomo, M. Dell'Aglio, F. Colao, R. Fantoni, and V. Lazic, Appl. Surf. Sci. 247, 157 (2005).

${ }^{4}$ V. Lazic, F. Colao, R. Fantoni, and V. Spizzicchino, Spectrochim. Acta, Part B 60, 1002 (2005).

${ }^{5}$ V. Lazic, F. Colao, R. Fantoni, and V. Spizzicchino, Spectrochim. Acta, Part B 60, 1014 (2005).

${ }^{6}$ T. Nishi, T. Sakka, H. Oguchi, K. Fukami, and Y. H. Ogata, J. Electrochem. Soc. 155, F237 (2008).

${ }^{7}$ S. Guirado, F. J. Fortes, V. Lazic, and J. J. Laserna, Spectrochim. Acta, Part B 74, 137 (2012).

${ }^{8}$ F. Mafune, J. Kohno, Y. Takada, and T. Kondow, J. Phys. Chem. B 104, 9111 (2000).

${ }^{9}$ T. Tsuji, K. Iryo, N. Watanabe, and M. Tsuji, Appl. Surf. Sci. 202, 80 (2002).

${ }^{10}$ P. Peyre, R. Fabbro, P. Merrien, and H. P. Lieurade, Mater. Sci. Eng., A 210, 102 (1996).

${ }^{11}$ Y. Sano, M. Obata, T. Kubo, N. Mukai, M. Yoda, K. Masaki, and Y. Ochi, Mater. Sci. Eng., A 417, 334 (2006). 
${ }^{12}$ B. Thornton, T. Masamura, T. Takahashi, T. Ura, K. Ohki, and T. Sakka, in Proceedings of IEEE/MTS Oceans 2012, Hampton Roads, VA (2012), p. 1.

${ }^{13}$ B. Thornton, T. Takahashi, T. Sato, T. Sakka, A. Tamura, A. Matsumoto, T. Nozaki, T. Ohki, and K. Ohki, Deep-Sea Res., Part I 95, 20 (2015).

${ }^{14} \mathrm{~T}$. Masamura, B. Thornton, and T. Ura, in Proceedings of MTS/IEEE Oceans 2011, Kona, HI (2011), p. 1.

${ }^{15}$ B. Thornton and T. Ura, Appl. Phys. Express 4, 022702 (2011).

${ }^{16}$ B. Thornton, T. Takahashi, T. Ura, and T. Sakka, Appl. Phys. Express 5, 102402 (2012).

${ }^{17}$ B. Thornton, T. Sakka, T. Takahashi, A. Tamura, T. Masamura, and A. Matsumoto, Appl. Phys. Express 6, 082401 (2013).

${ }^{18}$ B. Thornton, T. Sakka, T. Masamura, A. Tamura, T. Takahashi, and A. Matsumoto, Spectrochim. Acta, Part B 97, 7 (2014).

${ }^{19}$ K. Saito, K. Takatani, T. Sakka, and Y. H. Ogata, Appl. Surf. Sci. 197, 56 (2002).

${ }^{20}$ T. T. P. Nguyen, R. Tanabe, and Y. Ito, Appl. Phys. Lett. 102, 124103 (2013).

${ }^{21}$ T. Sakka, S. Iwanaga, Y. H. Ogata, A. Matsunawa, and T. Takemoto, J. Chem. Phys. 112, 8645 (2000).

${ }^{22}$ T. Sakka, K. Takatani, Y. H. Ogata, and M. Mabuchi, J. Phys. D 35, 65 (2002).

${ }^{23}$ R. Nyga and W. Neu, Opt. Lett. 18, 747 (1993).

${ }^{24}$ A. E. Pichahchy, D. A. Cremers, and M. J. Ferris, Spectrochim. Acta, Part B 52, 25 (1997).

${ }^{25}$ T. Sakka, A. Tamura, T. Nakajima, K. Fukami, and Y. H. Ogata, J. Chem. Phys. 136, 174201 (2012).

${ }^{26}$ A. De Giacomo, A. De Bonis, M. Dell'Aglio, O. De Pascale, R. Gaudiuso, S. Orlando, A. Santagata, G. S. Senesi, F. Taccogna, and R. Teghil, J. Phys. Chem. C 115, 5123 (2011).
${ }^{27}$ T. Takahashi, B. Thornton, and T. Ura, Appl. Phys. Express 6, 042403 (2013).

${ }^{28}$ T. Sakka, H. Oguchi, S. Masai, K. Hirata, Y. H. Ogata, M. Saeki, and H. Ohba, Appl. Phys. Lett. 88, 061120 (2006).

${ }^{29}$ T. Sakka, A. Tamura, A. Matsumoto, K. Fukami, N. Nishi, and B. Thornton, Spectrochim. Acta, Part B 97, 94 (2014).

${ }^{30}$ M. Dell'Aglio, R. Gaudiuso, O. De Pascale, and A. De Giacomo, "Mechanisms and processes of pulsed laser ablation in liquids during nanoparticle production," Appl. Surf. Sci. (published online).

${ }^{31}$ V. Lazic, R. Barbini, F. Colao, R. Fantoni, and A. Palucci, Spectrochim. Acta, Part B 56, 807 (2001).

${ }^{32}$ D. Bulajic, M. Corsi, G. Cristoforetti, S. Legnaioli, V. Palleschi, A. Salvetti, and E. Tognoni, Spectrochim. Acta, Part B 57, 339 (2002).

${ }^{33}$ T. Sakka, T. Nakajima, and Y. H. Ogata, J. Appl. Phys. 92, 2296 (2002).

${ }^{34}$ A. Matsumoto, A. Tamura, K. Fukami, Y. H. Ogata, and T. Sakka, Anal. Chem. 85, 3807 (2013).

${ }^{35}$ See supplementary material at http://dx.doi.org/10.1063/1.4919729 for the intensity profiles at the gate timings $\mathrm{A}$ and $\mathrm{C}$.

${ }^{36}$ T. Sakka, S. Masai, K. Fukami, and Y. H. Ogata, Spectrochim. Acta, Part B 64, 981 (2009).

${ }^{37}$ S. Ibrahimkutty, P. Wagener, A. Menzel, A. Plech, and S. Barcikowski, Appl. Phys. Lett. 101, 103104 (2012).

${ }^{38}$ A. Matsumoto, A. Tamura, K. Fukami, Y. H. Ogata, and T. Sakka, J. Appl. Phys. 113, 053302 (2013).

${ }^{39}$ J. Lam, D. Amans, F. Chaput, M. Diouf, G. Ledoux, N. Mary, K. Masenelli-Varlot, V. Motto-Ros, and C. Dujardin, Phys. Chem. Chem. Phys. 16, 963 (2014).

${ }^{40}$ O. R. Musaev, M. S. Driver, E. A. Sutter, A. N. Caruso, J. M. Wrobel, and M. B. Kruger, Appl. Phys. A 113, 355 (2013). 